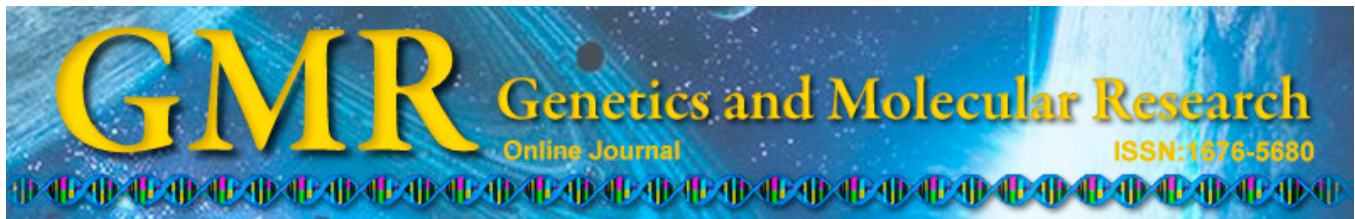

\title{
Loss of $\boldsymbol{S T A G} \mathbf{2}$ causes aneuploidy in normal human bladder cells
}

\author{
X. Li ${ }^{1,2 *}$, T.W. Zhang ${ }^{1 *}$, J.L. Tang ${ }^{2}$, P.P. Fa ${ }^{4}$, J.X. Lu ${ }^{2}$, F.M. Qi ${ }^{2}$, Z.M. Cai ${ }^{3}$, \\ C.X. Liu ${ }^{1}$ and X.J. Sun ${ }^{2,3}$ \\ ${ }^{1}$ Department of Urology, Zhujiang Hospital, \\ Second Affiliated Hospital of Southern Medical University, Guangzhou, \\ Guangdong Province, China \\ ${ }^{2}$ Shenzhen Tumor Immuno-Gene Therapy Clinical Application Engineering \\ Laboratory, Biobank of Shenzhen Second People's Hospital, \\ First Affiliated Hospital of Shenzhen University, Shenzhen, Guangdong Province, \\ China \\ ${ }^{3}$ National-Regional Key Technology Engineering Laboratory for Clinical \\ Application of Cancer Genomics, Shenzhen Second People's Hospital, \\ The First Affiliated Hospital of Shenzhen University, Shenzhen, \\ Guangdong Province, China \\ ${ }^{4}$ Shenzhen PKU-HKUST Medical Center, \\ Male Reproductive Medicine and Genetics, Institute of Urology, \\ Peking University Shenzhen Hospital, Shenzhen, Guangdong Province, China \\ *These authors contributed equally to this study. \\ Corresponding authors: C.X. Liu / X.J. Sun \\ E-mail: liuchx888@hotmail.com / xiaojuan26@gmail.com
}

Genet. Mol. Res. 14 (1): 2638-2646 (2015)

Received March 7, 2014

Accepted September 24, 2014

Published March 30, 2015

DOI http://dx.doi.org/10.4238/2015.March.30.24

ABSTRACT. The aim of this study was to determine how the function
of human stromal antigen 2 (STAG2) plays an important role in proper
chromosome separation. STAG2 mRNA in normal bladder cells and
bladder tumor cells was evaluated by RT-PCR. The protein levels of
STAG2 in normal bladder cells and bladder tumor cells were determined 
by western blot. A cell proliferation assay was used to measure the growth of tumor cells and STAG2-inhibited normal cells, and STAG2inhibited normal cells were subjected to karyotype analysis. Both STAG-2 mRNA and protein expression levels were lower in bladder cancer cells compared to the controls. Knockdown of STAG2 caused aneuploidy in normal bladder cells, leading to a decreased expression of the cohesin complex components SMC1, SMC3 and RAD21, but there was no obvious effect of STAG2 knockdown on cell proliferation. Our study indicated that abnormal expression of STAG2 could cause aneuploidy in normal bladder cells.

Key words: Urologic neoplasms; Stromal antigen 2; Aneuploidy; Bladder cancer; Cohesin complex

\section{INTRODUCTION}

Bladder cancer is one of the most common cancers worldwide, with transitional cell carcinoma (TCC) being the predominant form of this cancer. Our laboratory has previously reported a genomic analysis of TCC by both whole-genome and whole-exome sequencing of 99 individuals with TCC. A total of $32 \%$ of the samples harbored genetic alterations in the sister chromatid cohesion and segregation process. The mutation rate of stromal antigen 2 (STAG2), involved in the sister chromatid cohesion and segregation process, was 11\% (Guo et al., 2013).

STAG2 is a subunit of the cohesin complex, which comprises four core protein subunits that are conserved from yeast to vertebrates (Guacci et al., 1997; Michaelis et al., 1997; Darwiche et al., 1999). In human mitotic cells, the cohesin complex consists of RAD21, SMC1 $\alpha$, SMC3, and two SCC3 orthologues, SA1 and SA2 (Losada et al., 2000; Sumara et al., 2000). The structure of the cohesin complex has been well characterized; there are two major models of cohesin binding to capture DNA and to form a cohesion complex, namely the ring (Gruber et al., 2003) and handcuff (Zhang et al., 2008; Zhang and Pati, 2009) models. Its best known function is tethering the sister DNA molecules and holding sister chromatids together (Wong, 2010).

STAG2 is a new gene, which comprises 34 exons and has 4 types of mRNA, which has recently been of interest to scientists. STAG2 encodes SA2, a 141-kDa protein. The majority of previous studies of the function of STAG2 have concentrated primarily on the role of STAG2 in the cohesion complex, with STAG2 treated in these studies as part of SCC3 function (Losada et al., 2000). Studies have focused on investigating the role of SCC3 in the cohesin complex in mitosis (Sumara et al., 2000). It has been reported that SA2 is the primary cohesin in mammalian cells and that both SA1 and SA2 localize to the nucleus (Losada et al., 2000). Further research has shown that SA1 is required for telomere cohesion, whereas SA2 is uniquely required for centromere cohesion (Canudas and Smith, 2009). With growing interest in RAD21, as the closely interrelated partner, it has been reported that STAG2 and RAD21 are involved in several meiosis stages and cooperate with a STAG3-specific complex to maintain sister chromatid cohesion during the diplotene stage of meiosis (Prieto et al., 2002). Further research shows that the mutations acting at the RAD21 interface with the other cohesin proteins, namely SA2 and SMC1A, impair cellular DNA damage response, and disrupt transcription (Deardorff et al., 2012). In addition, SA2 has direct contacts with specific regions of the C-terminal end of CTCF, and all other cohesin components are recruited through their 
interaction with SA2 (Xiao et al., 2011). In 2011, Waldman's group showed that targeted inactivation of STAG2 led to chromatid cohesion defects and aneuploidy (Solomon et al., 2011). Subsequently, the loss of expression of STAG2 has been reported in colorectal, gastric and prostate carcinomas; however there are few mutations in STAG2 in these carcinomas (Kim et al., 2012). Similarly, somatic mutation of STAG2 is rare in acute leukemias (Chung et al., 2012) and neuroblastoma tumors (Djos et al., 2013). In contrast, in myeloid neoplasms, recurrent mutations and deletions have been detected in another study (Kon et al., 2013). Although there is dispute concerning the mutation of STAG2 in other carcinomas, it is reported by many scientists that there is a frequent mutation of STAG2 in bladder cancer (Solomon et al., 2011, 2013; Balbás-Martínez et al., 2013). However, there is currently no evidence that shows whether loss of STAG2 leads to aneuploidy in bladder cells.

The aim of the present study was to test the importance of the function of STAG2 in chromosome separation. Accordingly, STAG2 mRNA was determined in normal bladder and bladder tumor cells using real time RT-PCR, STAG2 protein levels in the normal and tumor cells were determined by Western blot, and the karyotype of STAG2-inhibited normal cells was analyzed.

\section{MATERIAL AND METHODS}

\section{Cell lines}

A panel of 3 bladder cancer cell lines (SW780, 5637 and T24) and the normal bladder cell line SV-HUC-1 were obtained from the American Type Culture Collection.

\section{Antibodies}

The following antibodies were used in this study: rabbit anti-SMC1 (Abcam, UK), anti-SMC3 and anti-RAD21 (Cell Signaling, USA), mouse anti-STAG2 (sc-81852, Santa Cruz Biotechnology, USA), and rabbit anti-GAPDH (Earthox, USA).

\section{Transfection}

Cells were seeded on standard plates and grown in culture medium for $8 \mathrm{~h}$ until they reached $30 \sim 50 \%$ confluence. Cells were transfected with siRNA by using Lipofectamin 2000 (Invitrogen, USA) according to manufacturer instructions. Based on the surface area of the plate, $0.25 \mu \mathrm{L}$ Lipofectamin 2000 and $10 \mathrm{pM}$ siRNA were used per square centimeter. A total of $25 \mu \mathrm{L}$ RPMI 1640 were used per square centimeter when making Lipofectamin 2000 siRNA complexes. The protein was collected at $48 \mathrm{~h}$ after transfection. Negative control and STAG2 siRNA were purchased from GenePharma (China). For double-siRNA experiments, each oligo was present at $20 \mathrm{nM}$. A quarter of the final double-siRNA was STAG2-1, a quarter was STAG2-2 and last half was STAG2-3 (siRNA oligo sequences are given in Table 1), which is the best protocol according to a pilot experiment. 
Table 1. siRNA oligo sequences used for transfection.

\begin{tabular}{ll}
\hline siRNA & Sequence (5' to 3') \\
\hline STAG-2-1 & F: GGU GAA GUA AGA CUC AAA UTT \\
STAG-2-2 & R: AUU UGA GUC UUA CUU CAC CTT \\
STAG-2-3 & F: GAG CCA UCC ACC UUU AAA UTT \\
& R: AUU UAA AGG UGG AUG GCU CTT \\
& F: GGA GGA UUG AGG AUC UUA ATT \\
\hline
\end{tabular}

\section{Western blot}

Cells were grown after transfection for $48 \mathrm{~h}$ and harvested in RIPA buffer. Protein was isolated from cancer cell lines in RIPA buffer on ice for $15 \mathrm{~min}$. Protein samples ( $30 \mu \mathrm{g} / \mathrm{lane})$ were then resolved by $10 \%$ SDS-PAGE. Electrophoresis was followed by blotting onto PVDF membranes, and the membranes were then incubated with the primary antibody at $4{ }^{\circ} \mathrm{C}$ overnight. The membrane was incubated with secondary antibody for $1 \mathrm{~h}$ at room temperature and exposed using WesternBright ECL-spray Western blotting detection system (Advansta, USA).

\section{Karyotyping}

Cultured cells were treated with $0.02 \mu \mathrm{g} / \mathrm{mL}$ colcemid for $55 \mathrm{~min}$ at $37^{\circ} \mathrm{C}$. The cells were then trypsinized, centrifuged for $7 \mathrm{~min}$ at $200 \mathrm{~g}$, and the cell pellet resuspended in warm hypotonic solution and incubated at $37^{\circ} \mathrm{C}$ for $11 \mathrm{~min}$. The swollen cells were centrifuged and the pellet resuspended in $8 \mathrm{~mL}$ Carnoy's fixative (3:1 methanol:glacial acetic acid). After incubation in fixative at room temperature for $96 \mathrm{~min}$, the cell suspension was centrifuged and washed twice in Carnoy's fixative. After the last centrifugation, the cells were resuspended in 1 to $3 \mathrm{~mL}$ freshly prepared fixative to produce an opalescent cell suspension. Drops of the final cell suspension were placed on clean slides and air-dried. Slides were stained with a 1:3 mixture of Wright's stain and 0.06 M phosphate buffer for 4-10 min, washed with tap water for $5 \mathrm{~s}$, and then air-dried. One hundred cells in metaphase were examined for chromosome count.

\section{Real-time RT-PCR}

Total RNA was extracted from cell lines using the RNeasy Mini kit (QIAGEN, Germany) according to the manufacturer protocol. The PrimeScript RT reagent kit with gDNA Eraser (Takara, Japan) was used to synthesize the first-strand cDNA. The total reaction volume was $20 \mu \mathrm{L}$ including $500 \mathrm{ng} \mathrm{RNA}$, and the reaction mixture was incubated at $37^{\circ} \mathrm{C}$ for 15 $\mathrm{min}$, heated at $55^{\circ} \mathrm{C}$ for $5 \mathrm{~s}$ and then cooled on ice.

The primers were designed by Primer 5, on the basis of their mRNA sequences. The corresponding primer sequences are listed in Table 2. Real-time PCR was carried out with SYBR Green dye in SYBR Premix Ex Taq $\alpha$ (Takara). The 50- $\mu \mathrm{L}$ real-time PCR mixture contained 1 $\mu \mathrm{L}$ cDNA (synthesized as described above), $25 \mu \mathrm{L}$ SYBR Green master mix, $1 \mu \mathrm{L}$ Rox reference dye and $2 \mu \mathrm{L}$ of each upstream and downstream primer. The amplification conditions were $95^{\circ} \mathrm{C}$ for $30 \mathrm{~s}$ followed by 40 cycles of $95^{\circ} \mathrm{C}$ for $5 \mathrm{~s}$ and $60^{\circ} \mathrm{C}$ for $30 \mathrm{~s}$. Relative expression levels of the target genes were normalized to the geometric mean of the internal control gene, GAPDH. The data were analyzed using the comparative threshold cycle $\left(2^{-\Delta C T}\right)$ method. 
Table 2. Primer oligo sequences used for Real time PCR.

\begin{tabular}{ll}
\hline Primer & Sequence (5' to 3') $^{\prime}$ \\
\hline STAG-2 & F: ACGGAAAGTGGTTGAGGG \\
& R: GTGGAGGTGAGTTGTGGTGT \\
SMC1A & F: GTGGACTCGGAAGACAGGC \\
& R: GGCTCATAGCGAATCACATCA \\
SMC1B & F: GGCAAGAGGGCACATGAAGTA \\
& R: ACAGCC CACAAACAGAAAGGA \\
SMC3 & F: GATGAAGGAGAAGGGAGTGGT \\
& R: : ATCAGAGCAAGGGCTACCAAG \\
RAD21 & F: :TACTGAAGCTCTTTACACGCTGTC \\
GAPDH & R: : ATGCTGCTGTTGCTGGTCCTC \\
& F: AGCAAGAGCACAAGAGGAAGAG \\
& R: TCTACATGGCAACTGTGAGGAG \\
\hline
\end{tabular}

\section{RESULTS}

\section{Significantly higher STAG2 mRNA and protein levels in normal bladder cells than bladder cancer cells}

Western blot analysis was used to measure STAG2 protein levels in normal human bladder cells (SV-HUC-1) and human bladder tumor cells (T24, SW780, and 5637), and their gamma ratios were analyzed (Figure 1A). STAG2 protein levels were significantly higher (from 2.5 to 4.2 times, $\mathrm{N}=3, \mathrm{P}<0.05$ ) in normal bladder cells than bladder cancer cells, similar to mRNA levels (Figure 1B). Real-time RT-PCR showed that STAG2 mRNA was significantly higher (nearly 10 times) in normal bladder cells than bladder tumor cells $(\mathrm{N}=3, \mathrm{P}$ $<0.05$ ) (Figure 1C). Since SW780 cells represented the lowest tumor stage and T24 cells the highest, loss of STAG2 in these cells was likely to be associated with low tumor stage, which is consistent with conclusions drawn by Taylor et al. (2013).

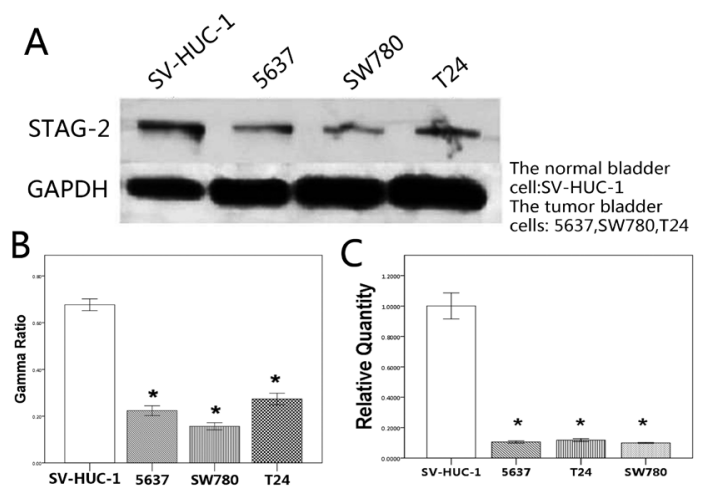

Figure 1. A. Western blot of STAG-2 protein expression in normal human bladder cell (SV-HUC-1) and 3 bladder cancer cell lines $(T 24,5637$, and SW780). The protein expression of STAG-2 in normal cells was higher than in the bladder tumor cell lines. B. The gamma ratios analysis showed the protein expression of STAG-2 in normal cells was higher than bladder tumor cells $(\mathrm{N}=3, \mathrm{P}<0.05)$. C. Real time PCR of STAG-2 mRNA in normal human bladder cells (SV-HUC-1) and 3 bladder cancer cell lines $(T 24,5637$, and SW780). The mRNA of STAG-2 in normal bladder cells was much higher than in the bladder tumor cell lines $(5637, T 24, S W 780)(\mathrm{N}=3, \mathrm{P}<0.05)$. 


\section{Knockdown of STAG2 leads to aneuploidy in normal bladder cells}

Inhibition of STAG2 expression by siRNA in SV-HUC-1 cells, half near diploid and half near tetraploid human cells with stable karyotypes, which are usually regarded as normal control, led to aneuploidy as determined by karyotype analysis. Two representative illustrations are shown to indicate that inhibition of STAG2 resulted in stable karyotypes becoming unstable karyotypes with random changes in chromosome number (Figure 2A,B). The control cells have 44 chromosomes and lack number 15 chromosomes (Figure 2A). In STAG2-inhibited cells, there were monosomies of chromosomes 5, 6, 10, and 12, trisomies of chromosomes $2,16,20$, and 21, and tetrasomy of chromosome 22 (Figure 2B). The chromosome numbers of 100 cells were determined in the STAG2 knockdown group and control group. There was a significant difference between the two groups (Figure 2C) $(\mathrm{P}=0.012)$.

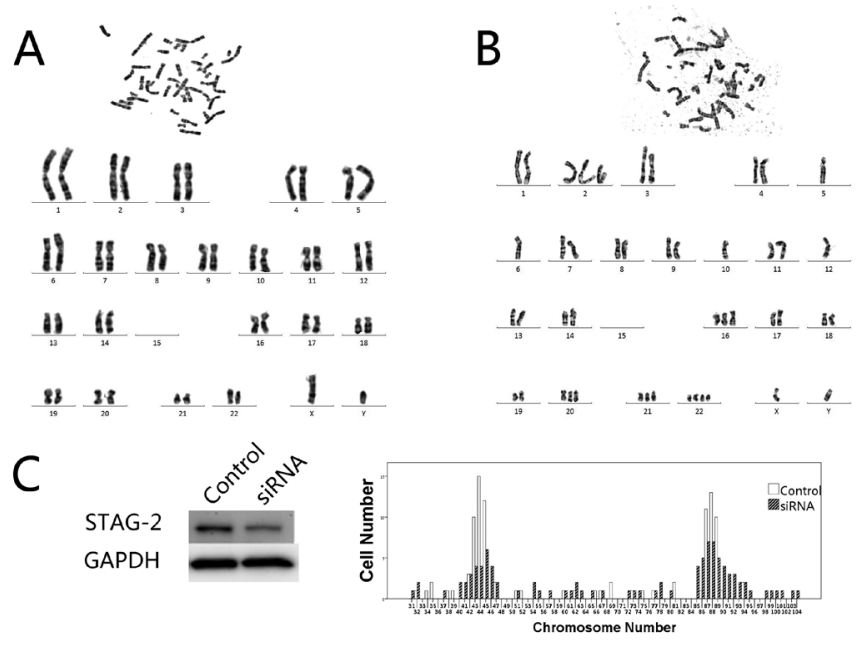

Figure 2. Two representative karyotypes from normal bladder cells (SV-HUC-1). A. Control normal cells were chosen to show control karyotype in the control cells, which lack of No.15 chromosomes. B. STAG-2-inhibited cells were chosen to show aneuploidy in these cells. These karyotypes contained obvious chromosomal aberrations including monosomies, trisomies, and tetrasomies. C. The chromosome number of STAG-2-inhibited cells was significantly different from the control cells $(\mathrm{P}=0.012)$.

\section{Inhibition of STAG2 results in lower expression of SMC1, SMC3, and RAD21}

Real-time RT-PCR was used to measure the mRNA expression of SMC1A, SMC1B, SMC3, and RAD21, following STAG2 knockdown with siRNA in normal bladder cells (Figure 3). mRNA levels of SMC1, SMC1B, SMC3, and RAD21 in the STAG2-inhibited group were significantly lower than in the control group $(\mathrm{N}=3, \mathrm{P}<0.05)$, and the protein levels were similar to the mRNA levels. The gamma ratios were analyzed, and the cohesin components were found to be reduced by nearly half when STAG2 expression was decreased by approximately half (Figure 4) $(\mathrm{N}=3, \mathrm{P}<0.05)$. 


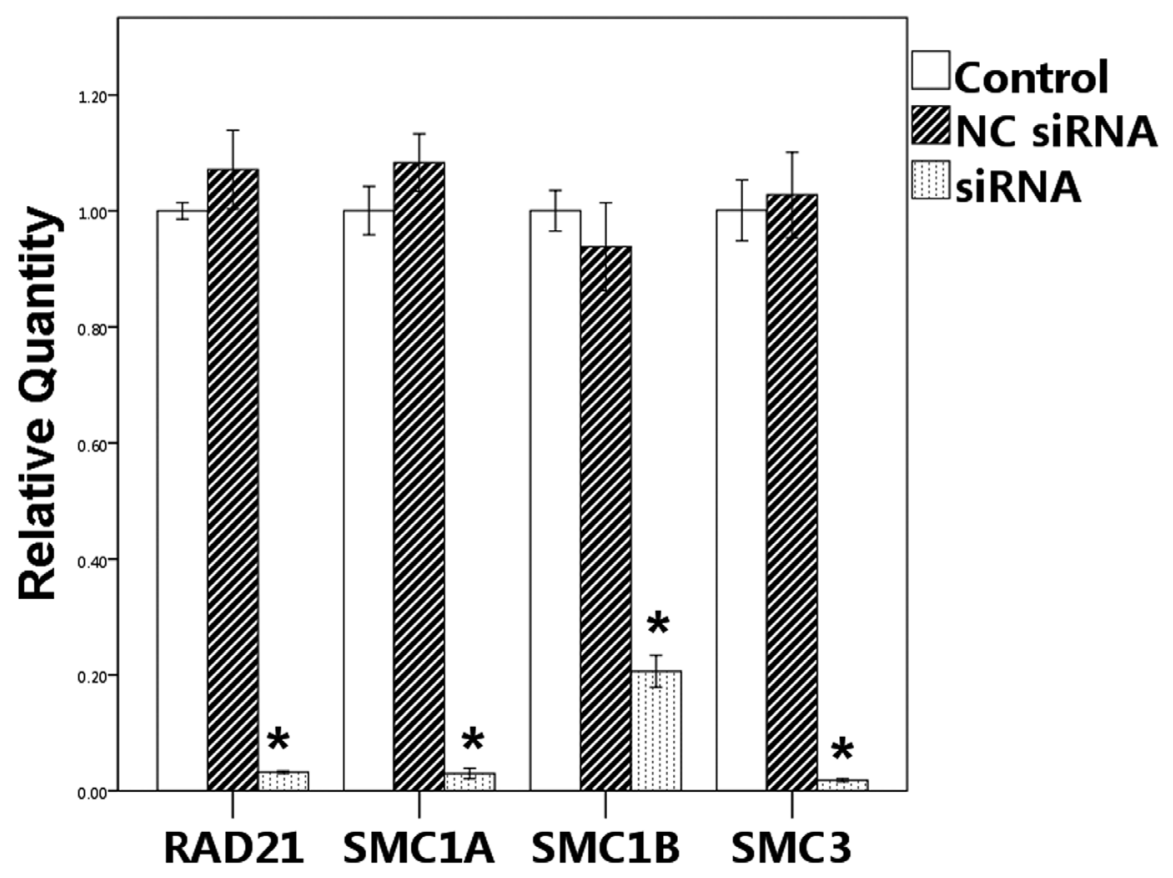

Figure 3. Real-time PCR of the mRNA levels of SMC1A, SMC1B, RAD21, and SMC3 in normal control bladder cells, normal bladder cells transfected with control RNA and normal bladder cells transfected with STAG-2 siRNA showed that when STAG-2 was knocked down, mRNA levels significantly decreased simultaneously $\left(\mathrm{N}=3,{ }^{*} \mathrm{P}<0.05\right)$.

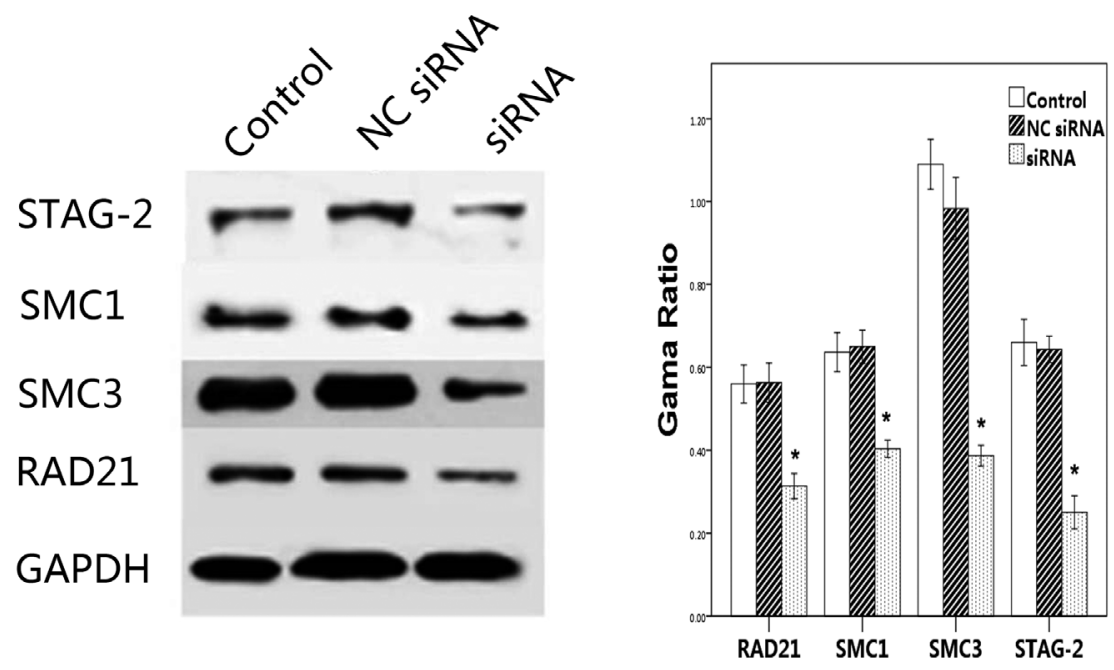

Figure 4. Western blot analysis of the expression of STAG-2, and the cohesion components SMC1, SMC3, and RAD21 in normal control bladder cells, normal bladder cells transfected with normal RNA and normal bladder cells transfected with STAG-2 siRNA. When compared to the housekeeping gene GAPDH with gamma ratio analysis, protein levels of SMC1, SMC3, and RAD21 were significantly decreased in the STAG-2-inhibited normal bladder cells $(\mathrm{N}=3, \mathrm{P}<0.05)$. 


\section{DISCUSSION}

The cohesin complex, which is essential to ensure successful chromosome separation, was first described in the mid-1990s (Guacci et al., 1997; Michaelis et al., 1997; Losada et al., 2000). The cohesin complex consists of SMC1, SMC3, RAD21 and two SCC3 orthologues, SA1 and SA2 (Losada et al., 2000; Sumara et al., 2000). Its best-known function is tethering the sister DNA molecules and holding sister chromatids together. Further research has indicated that SA1 is required for telomere cohesion, whereas SA2 is uniquely required for cohesion at centromeres (Canudas and Smith, 2009).

In the current study, knocking down STAG-2 led to lower mRNA and protein levels of SMC1, SMC3, and RAD21, which are the other subunits of the cohesin complex. This suggests that inhibition of STAG2 leads to loss of the cohesin complex, which may be the centromere cohesin, thus decreasing the function of the cohesin complex. Since the cohesin complex works as a whole, loss of STAG2 expression causes a relative increase in SMC1, SMC3, and RAD21. There may also be a nonspecific feedback that causes a decrease in other subunits to decrease the cohesin complex.

In a previous study, the protein shugoshin was shown to protect the centromere cohesin from phosphorylation and removal (Kitajima et al., 2004; Salic et al., 2004). Depletion of shugoshin results in premature separation of sister chromatids and aneuploidy in human colon cancer cells (Iwaizumi et al., 2009). It could be conjectured that removal or lack of centromere cohesin causes aneuploidy. In the current study, the loss of STAG2 expression led the normal bladder cells into aneuploidy and also decreased the cohesin complex, where the lack of centromere cohesin probably caused aneuploidy.

Meanwhile, Balbás-Martínez et al. (2013) suggested that knocking down STAG2 in bladder cancer cells did not increase aneuploidy. As seen by determining chromosome number, knocking down STAG2 in bladder cancer cells may affect the degree of aneuploidy, such as more monosomies, trisomies and so on, and result in little offset of chromosome peak to show a statistical difference. It is also possible that STAG2 plays a different role in genetically unstable cells.

\section{ACKNOWLEDGMENTS}

Research supported the National Nature Science Foundation of China (\#NSFC 81101584 \#\&81372507), the International S\&T Cooperation Program of China (\#2014DFA31050), the Shenzhen Peacock Foundation (\#KQC201106020032A), the Shenzhen Basic Research Key Project (\#JCYJ20120614455816670), the Development Program for Basic Research of China "973" Program (\#2014CB745200), and the Chinese High-Tech “863” Program (\#2012AA02A201 \& 2012AA02A208).

\section{REFERENCES}

Balbás-Martínez C, Sagrera A, Carrillo-de-Santa-Pau E, Earl J, et al. (2013). Recurrent inactivation of STAG2 in bladder cancer is not associated with aneuploidy. Nat. Genet. 45: 1464-1469.

Canudas S and Smith S (2009). Differential regulation of telomere and centromere cohesion by the Scc3 homologues SA1 and SA2, respectively, in human cells. J. Cell Biol. 187: 165-173.

Chung NG, Kim MS, Yoo NJ and Lee SH (2012). Somatic mutation of STAG2, an aneuploidy-related gene, is rare in acute leukemias. Leuk. Lymphoma 53: 1234-1235. 
Darwiche N, Freeman LA and Strunnikov A (1999). Characterization of the components of the putative mammalian sister chromatid cohesion complex. Gene 233: 39-47.

Deardorff MA, Wilde JJ, Albrecht M, Dickinson E, et al. (2012). RAD21 mutations cause a human cohesinopathy. Am. J. Hum. Genet. 90: 1014-1027.

Djos A, Fransson S, Kogner P and Martinsson T (2013). Aneuploidy in neuroblastoma tumors is not associated with inactivating point mutations in the STAG2 gene. BMC Med. Genet. 14: 102.

Gruber S, Haering CH and Nasmyth K (2003). Chromosomal cohesin forms a ring. Cell 112: 765-777.

Guacci V, Koshland D and Strunnikov A (1997). A direct link between sister chromatid cohesion and chromosome condensation revealed through the analysis of MCD1 in S, cerevisiae. Cell 91: 47 -57.

Guo G, Sun X, Chen C, Wu S, et al. (2013). Whole-genome and whole-exome sequencing of bladder cancer identifies frequent alterations in genes involved in sister chromatid cohesion and segregation. Nat. Genet. 45: 1459-1463.

Iwaizumi M, Shinmura K, Mori H, Yamada H, et al. (2009). Human Sgol downregulation leads to chromosomal instability in colorectal cancer. Gut 58: 249-260.

Kim MS, Kim SS, Je EM, Yoo NJ, et al. (2012). Mutational and expressional analyses of STAG2 gene in solid cancers. Neoplasma 59: 524-529.

Kitajima TS, Kawashima SA and Watanabe Y (2004). The conserved kinetochore protein shugoshin protects centromeric cohesion during meiosis. Nature 427: 510-517.

Kon A, Shih LY, Minamino M, Sanada M, et al. (2013). Recurrent mutations in multiple components of the cohesin complex in myeloid neoplasms. Nat. Genet. 45: 1232-1237.

Losada A, Yokochi T, Kobayashi R and Hirano T (2000). Identification and characterization of SA/Scc3p subunits in the Xenopus and human cohesin complexes. J. Cell Biol. 150: 405-416.

Michaelis C, Ciosk R and Nasmyth K (1997). Cohesins: chromosomal proteins that prevent premature separation of sister chromatids. Cell 91: 35-45.

Prieto I, Pezzi N, Buesa JM, Kremer L, et al. (2002). STAG2 and Rad21 mammalian mitotic cohesins are implicated in meiosis. EMBO Rep. 3: 543-550.

Salic A, Waters JC and Mitchison TJ (2004). Vertebrate shugoshin links sister centromere cohesion and kinetochore microtubule stability in mitosis. Cell 118: 567-578.

Solomon DA, Kim T, Diaz-Martinez LA, Fair J, et al. (2011). Mutational inactivation of STAG2 causes aneuploidy in human cancer. Science 333: 1039-1043.

Solomon DA, Kim JS, Bondaruk J, Shariat SF, et al. (2013). Frequent truncating mutations of STAG2 in bladder cancer. Nat. Genet. 45: 1428-1430.

Sumara I, Vorlaufer E, Gieffers C, Peters BH, et al. (2000). Characterization of vertebrate cohesin complexes and their regulation in prophase. J. Cell Biol. 151: 749-762.

Taylor CF, Platt FM, Hurst CD, Thygesen HH, et al. (2013). Frequent inactivating mutations of STAG2 in bladder cancer are associated with low tumor grade and stage and inversely related to chromosomal copy number changes. Hum. Mol. Genet. [Epub ahead of print]

Wong RW (2010). An update on cohesin function as a 'molecular glue' on chromosomes and spindles. Cell Cycle 9: 1754-1758.

Xiao T, Wallace J and Felsenfeld G (2011). Specific sites in the C terminus of CTCF interact with the SA2 subunit of the cohesin complex and are required for cohesin-dependent insulation activity. Mol. Cell. Biol. 31: 2174-2183.

Zhang N and Pati D (2009). Handcuff for sisters: a new model for sister chromatid cohesion. Cell Cycle 8: 399-402.

Zhang N, Kuznetsov SG, Sharan SK, Li K, et al. (2008). A handcuff model for the cohesin complex. J. Cell Biol.183: 1019-1031. 